\title{
Percutaneous cholecystostomy: Case report and literature review
}

\author{
HARVEY M. WIENER, DO \\ Pittsburgh, Pennsylvania \\ JOSEPH D. FLYNN, JR., DO \\ Yardley, Pennsylvania
}

The performance of a percutaneous cholecystostomy in a community hospital setting to relieve obstructive jaundice in an 87-year-old patient with pancreatic head tumor is described. The procedure relieved the patient's obstructive symptoms and allowed him to be discharged to home care. Since first reported in 1878 , percutaneous cholecystostomy primarily has been a surgical procedure. With the evolution of interventional radiology, an increasing number of percutaneous procedures are being used. In the unstable patient, biliary decompression via percutaneous cholecystostomy is advocated until definitive surgery can be performed. Percutaneous cholecystostomy, under proper guidance, can be of beneficial use in the relief of obstructive jaundice.

The benefits versus the risks of percutaneous cholecystostomy has been a controversial topic in the radiologic and surgical literature. Complications such as severe vasovagal reactions, cholangitis peritonitis, sepsis, and death are well documented since the first planned cholecystostomy was performed by Sims in 1878. 1-3 The indications for cholecystostomy are varied: (1) drainage of benign or malignant obstruction; (2) decompression of hydrops; (3) chemodissolution of cholelithiasis, and (4) preoperative stabilization or palliation in the unstable patient. Yet, there is no clear consensus as to the indications or contraindications for this procedure..$^{5-7}$

Our case demonstrates the practicality of direct puncture and drainage of the gallbladder in a community hospital setting.

\section{Report of case}

An 87-year-old white man presented to our emergency department with a complaint of painless jaundice. The patient had acholic stools and dark urine. Histories of alcohol or drug abuse were denied. Physical examination revealed a right upper quadrant mass. A recent, outpatient ultrasound revealed a single cholelith and dilatation of the common bile duct. The total direct and indirect bilirubins as well as liver function parameters suggested obstructive jaundice.

Shortly after admission, the patient became encephalopathic and his condition deteriorated. A percutaneous chotangiogram was performed. Using a Medi-Tech onestick system, a percutaneous cholangiography was performed. It revealed a complete obstruction of the distal common bile duct. The inner surface of the obstruction was concave, suggesting a tumor (Fig. 1). The tract was then dilated, a 7Fr Cobra catheter, $\mathrm{C}-2$ curve was inserted over 0.035 Luderquist exchange guidewire, and the guidewire removed. Manipulation past the obstruction was not possible. Over a guidewire, the Cobra catheter was then replaced by a modified $10.2 \mathrm{Fr}$ Cope biliary drainage catheter. A later computed tomography scan of the pancreas demonstrated a tumor obstructing the common bile duct (Figs. 2 and 3 ).

The patient was doing well and his total bilirubin level was decreasing when he traumatically removed the catheter five days later. Reinsertion of a catheter was attempted. It was unsuccessful due to the decompressed biliary system. With a $10.2 \mathrm{~F}$ hole in the liver and bile ducts and a common bile duct obstruction, bile peritonitis became a growing concern. A percutaneous cholecystostomy was then performed on the same day as the traumatic catheter removal.

\section{Technique}

The skin and subcutaneous tissue overlying the right midaxillary line were infiltrated with Lidocaine (Fig. 4). Under fluoroscopic control a 19-gauge Lunderquist PTC sheath needle was introduced into the gallbladder fundus through the liver-gallbladder bed. After removing the needle stylus, a 0.035 Lunderquist exchange guidewire was inserted. An 8.3Fr Ring Biliary Drain- 


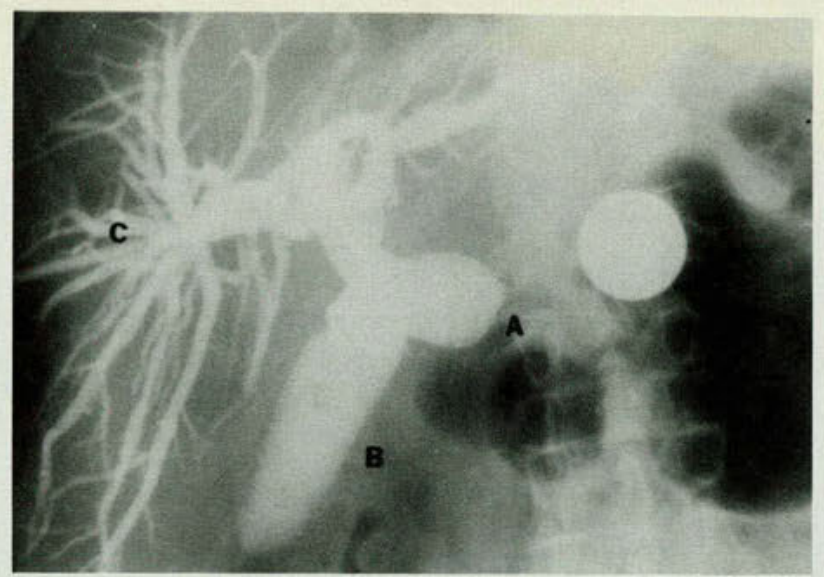

Fig. 1.Percutaneous cholangiogram demonstrates (A) obstructed and dilated common bile duct, $(B)$ contrast-filled gallbladder, and $(C)$ catheter. The metallic disk overlying the spine is a marker.

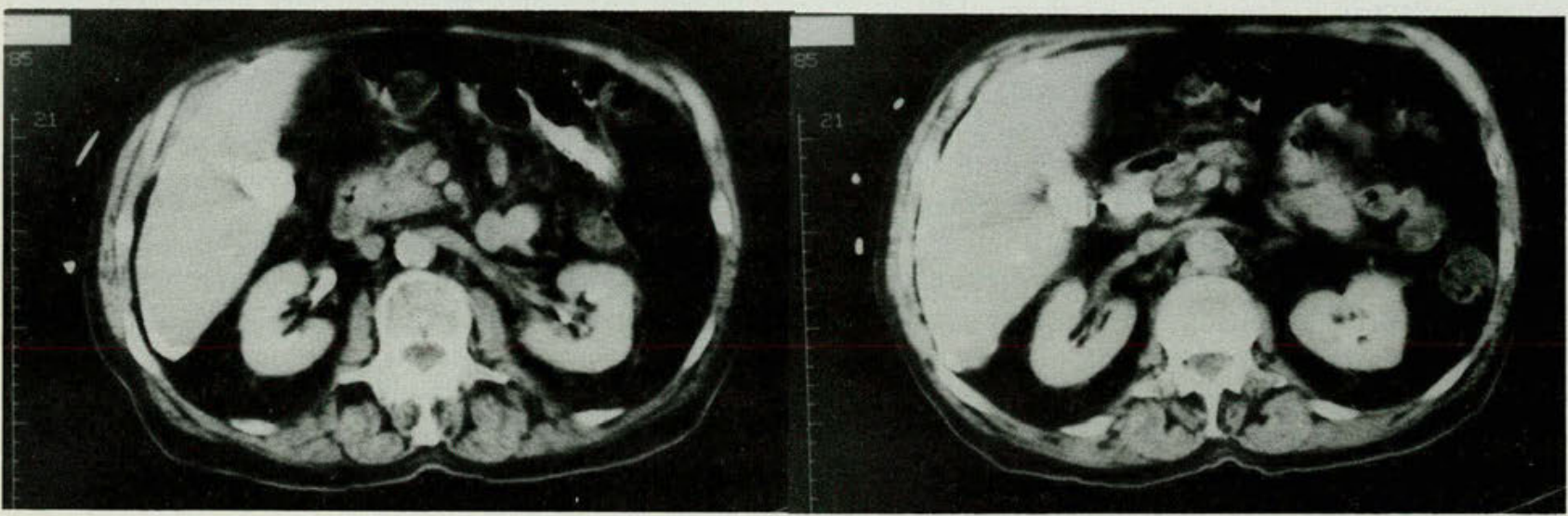

Figs. 2 and 3. Computed tomographic scans performed demonstrate a mass after percutaneous cholangiography in the head of the pancreas. Note the contrast-filled common bile duct.

age Catheter was placed over the wire, the tip was manipulated into the cystic duct. The wire was then removed. Injection of $43 \%$ water-soluble iodinated contrast medium through the biliary catheter revealed opacification of the gallbladder, cystic duct, common bile duct, and intrahepatic radicles (Fig. 5). No extravasation of contrast medium was seen.

\section{Clinical course}

The patient became septic after traumatic removal of the initial drainage catheter. Blood cultures grew group $\mathrm{D}$ streptococcus. The patient was treated with antibiotic therapy and fluids. Repeat blood cultures were negative.

After treatment of the sepsis and insertion of the percutaneous cholecystostomy catheter, the patient's condition stabilized and then improved. The total bilirubin decreased from $39 \mathrm{mg} / \mathrm{dL}$ (normal $0.3-1.2 \mathrm{mg} / \mathrm{dL}$ ) on admission to $5.4 \mathrm{mg} / \mathrm{dL}$ four days prior to discharge. By that time the patient was ambulating, performing self- care functions, and tolerating a regular diet. The patient was discharged to home care on the eighth postoperative day. Follow up was arranged through the department of surgery.

\section{Discussion}

The prevalence of biliary tract disease places operations on the biliary system among the most frequently performed intra-abdominal procedures. ${ }^{8,9}$ In the unstable patient, percutaneous cholecystostomy can be used for biliary decompression of bile and pus and/or contrast cholangiography. ${ }^{10}$

Many authors have advocated direct percutaneous gallbladder puncture, either under fluoroscopic or ultrasonic guidance. ${ }^{11-13}$ The most common complication of direct gallbladder puncture, intraperitoneal bile leakage, can be potentially serious. The severity of this complication can be diminished if 


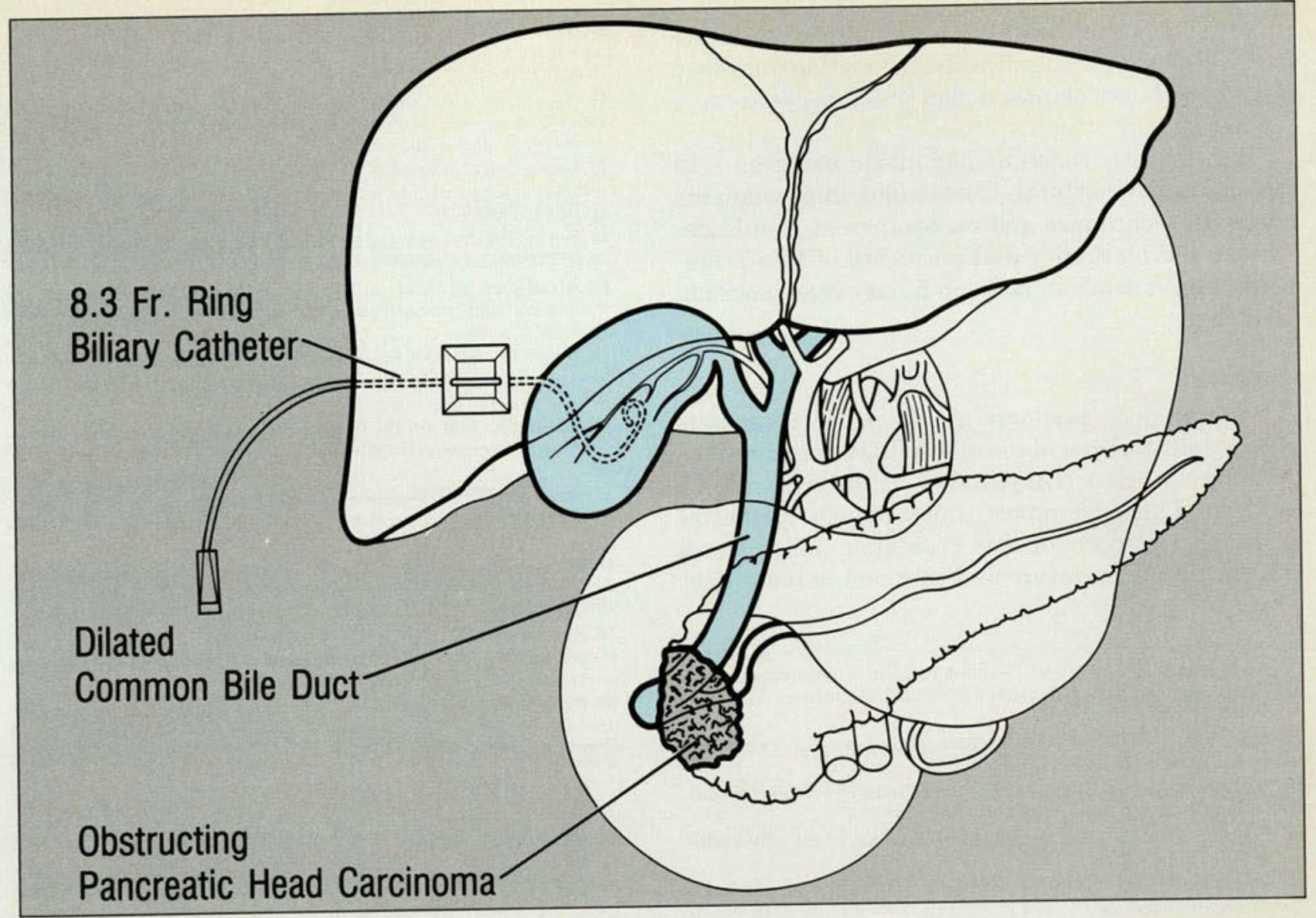

Fig. 4. Percutaneous cholecystostomy.

the bile is sterile, hardware manipulation is kept to a minimum, and the size of the puncture is kept as small as possible. The route of puncture is also important. Puncturing the gallbladder through the liver uses the liver parenchyma as an anchor for the catheter and a seal for the gallbladder puncture site. ${ }^{14-16}$

Percutaneous cholecystostomy does appear to have value in palliation of biliary obstruction in severely debilitated patients. Under careful guidance, the procedure is relatively simple to perform, especially if the gallbladder is large and situated near the abdominal wall. Ultrasonic guidance can increase the convenience of performing this procedure by conceivably limiting the number of punctures. It also suggests the possibility of performing a percutaneous cholecystostomy outside of the special procedures suite. ${ }^{17-20}$

Allen and associates ${ }^{6}$ have reported on chemodissolution of gallstones by methyltertbutyl ether via percutaneous cholecystostomy. Monooctanoin, another solubilizing agent, is also being used.

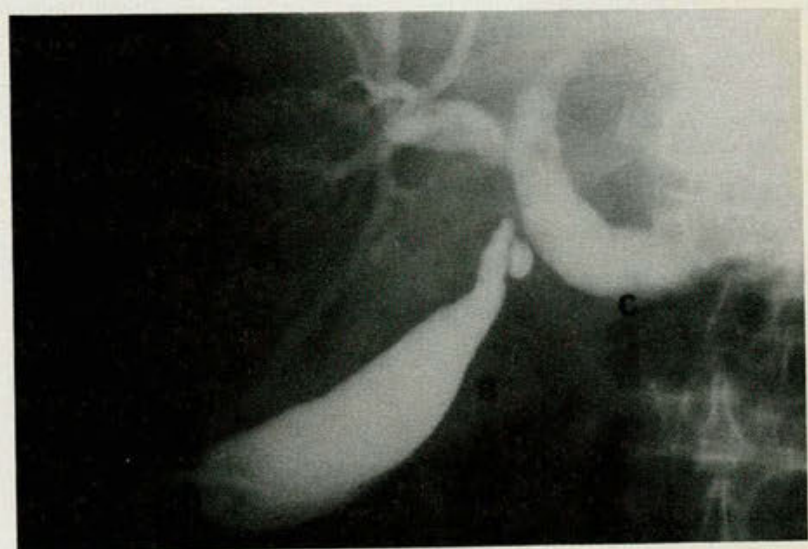

Fig. 5. Contrast cholangiogram depicts (A) the cholecystostomy catheter and opacification of $(B)$ the gallbladder and $(C)$ the common bile duct.

\section{Comment}

Although our patient was in poor condition, the procedure appeared to be safe and helpful in relieving the obstructive symptoms. Sepsis was the only complication - the result of the patient's trau- 
matic removal of the initial biliary drainage catheter. The patient's jaundice and mentation improved within 48 hours of insertion of the cholecystostomy catheter.

We are encouraged by our initial experience in a community hospital. Continuous improvements made in techniques and on equipment should decrease the morbidity and mortality of this procedure, and expand our scope in biliary interventional radiology.

\section{Summary}

Under proper radiologic guidance, percutaneous cholecystostomy is a simple palliative procedure to relieve obstructive jaundice in the critically ill patient. This case report illustrates the potential benefits and risks of this procedure and reviews the pertinent literature and opinions on its utility.

1. von Sonnenberg E, Wing VW, Pollard JW, et al: Life-threatening vagal reactions associated with percutaneous cholecystostomy. Radiology 1984;151:377-380.

2. Beart RW Jr, Mroz CT: Cholecystostomy for noninflammatory disease. Am J Surg 1981;141:342-343.

3. Skillings JC, Kumai C, Hinshaw JR: Cholecystostomy: A place in modern biliary surgery? Am J Surg 1980;139:865-869.

4. Sims JM: Remarks on cholecystostomy in dropsy of the gallbladder. Br Med $J$ 1878;11:811-815.

5. von Sonnenberg E, Wittich GR, Casola G, et al: Diagnostic and therapeutic percutaneous gallbladder procedures. Radiology 1986;160:23-26.

6. Allen MJ, Borody TJ, Bugliosi TF, et al: Rapid dissolution of gallstones by methyl tert-butyl ether: Preliminary observations. $N$ Engl $J$ Med 1985;312:217-220.

7. Havard C, Parry D: Cholecystostomy. Br J Surg 1976;63:631-636.

8. Glenn F: Cholecystostomy in the high-risk patient with biliary tract disease. Ann Surg 1977; 185:185-191.

9. Shaver RW, Hawkins IF Jr, Soong J: Percutaneous cholecystostomy AJR 1982;138:1133-1136.
10. Molnar W, Stockman AE: Relief of obstructive jaundice through percutaneous transhepatic catheter-a new therapeutic method AJR $1974 ; 122: 356-367$

11. Eggermont AM, Lameris JS, Jeekel J: Ultrasound-guided percutaneous transhepatic cholecystostomy for acute acalculous cholecystitis Arch Surg 1985;120:1354-1356.

12. Pearse DM, Hawkins IF, Jr, Shaver R, et al: Percutaneous cholecystostomy in acute cholecystitis and common duct obstruction. Radiol. ogy 1984;152:365-367.

13. Kerlan RK Jr, LaBerge JM, Ring EJ: Percutaneous cholecystolithotomy: Preliminary experience. Radiology 1985;157:653-656.

14. Elyaderani M, Gabriele OF: Percutaneous cholecystostomy and cholangiography in patients with obstructive jaundice. Radiology 1979;130:601-602.

15. Joseph PK, Bizer IS, Sprayregen SS, et al: Percutaneous transhepatic biliary drainage. Results and complications in 81 patients. JAMA 1986;255:2763-2767.

16. Teplick SK, Wolferth CC Jr, Hayes MF Jr, et al: Percutaneous cholecystostomy in obstructive jaundice. Gastrointestin Radiol 1982;7:259 261.

17. Longmaid HE III, Bassett JG, Gottlieb H: Management of gallbladder perforation by percutaneous cholecystomy. Crit Care Med 1985;13:686687.

18. Demasi CJ, Akdamar K, Sparks RD, et al: Puncture of the gallbladder during percutaneous transhepatic cholangiography. JAMA 1967;2011:79-82.

19. Elyaderani MK, McDowell DE, Gabriele OF: A preliminary report of percutaneous cholecystostomy under ultrasonography and fluoroscopy guidance. J Clin Gastroenterol 1983;5:277-281.

20. McGahan JP, Anderson MW, Walter JP: Portable real-time sonography and needle guidance systems for aspiration and drainage. Am $J$ Roentgenol 1986;147:1241-1246.

At the time this paper was written, Dr. Wiener was the chief radiology resident in the Medical Imaging Department, Kennedy Memorial Hospitals, University Medical Center, Stratford, NJ. Paul Chase, DO, is the residency program director and Milton Metzman, DO, is chairman of the Department of Radiology. Dr. Wiener is now a fellow in angiography and interventional radiology at Western Pennsylvania Hospital, Pittsburgh, Pa. Dr. Flynn is director, Special Studies Department, Delaware Valley Medical Center, Langhorne, $\mathrm{Pa}$.

Dr. Wiener, Western Pennsylvania Hospital, 4800 Friendship Ave, Pittsburgh, $\mathrm{Pa} 15224$. 


\section{"Deafness is something you put beside you not in front of you."}

LINDA BOVE / ACTRESS

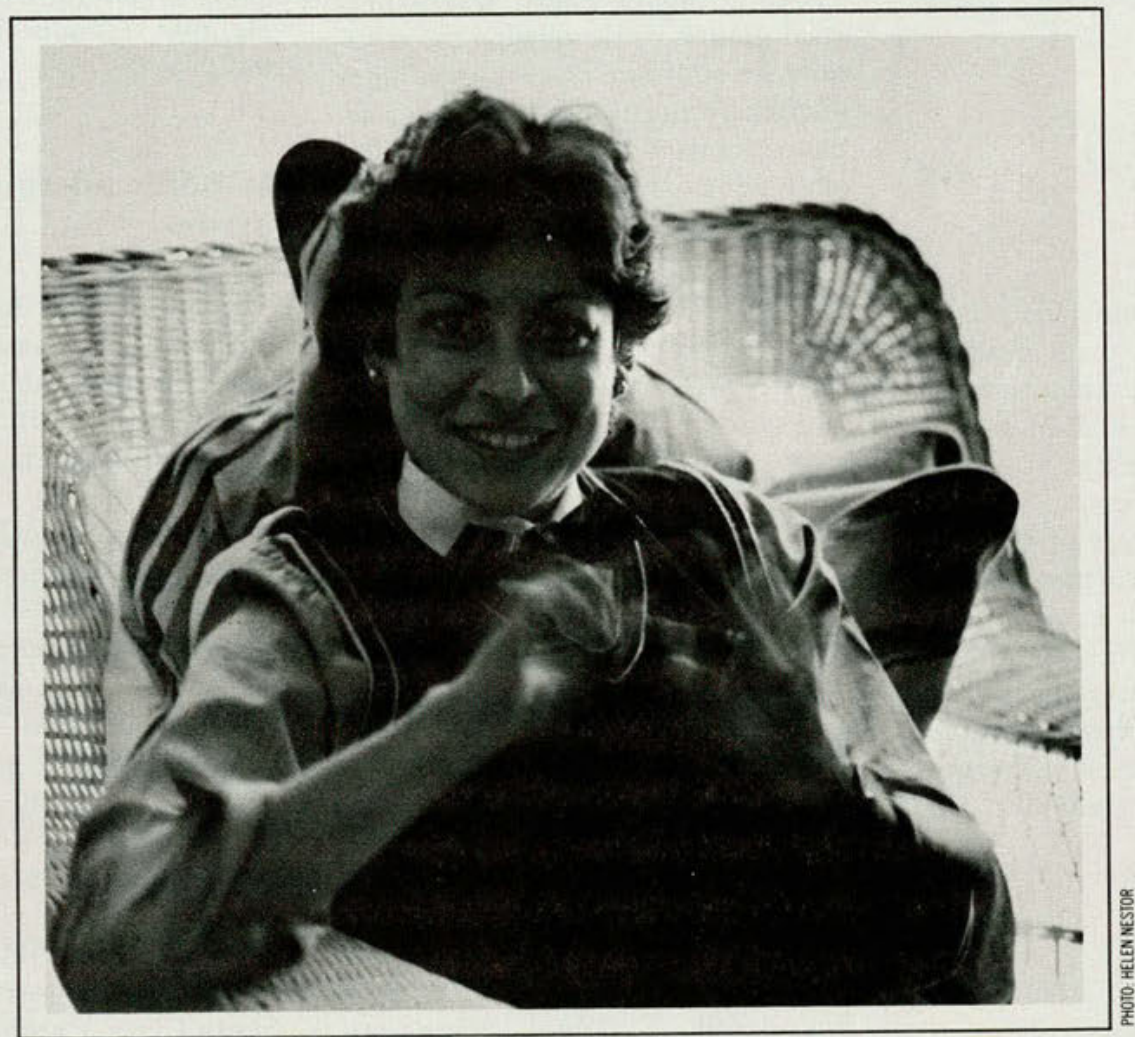

Linda Bove performed with The National Theatre of the Deaf for nine years. She has also starred in the Tony Award winning show, Children of a Lesser God.

\section{Believe in them. Break the barriers. PRESIDENT'S COMMITTEE ON EMPLOYMENT OF THE HANDICAPPED, WASHINGTON D.C. 20210}

